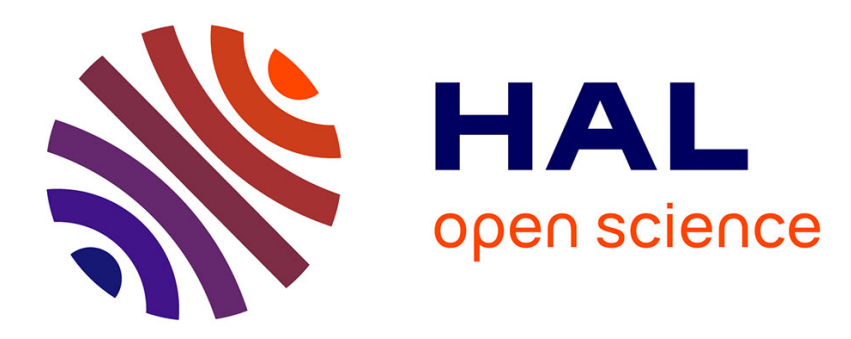

\title{
Tubes électroniques de grande puissance pour le chauffage des plasmas \\ P. Guenard, P. Palluel
}

\section{To cite this version:}

P. Guenard, P. Palluel. Tubes électroniques de grande puissance pour le chauffage des plasmas. Revue de Physique Appliquée, 1977, 12 (8), pp.1163-1170. 10.1051/rphysap:019770012080116300 . jpa-00244294

\section{HAL Id: jpa-00244294 https://hal.science/jpa-00244294}

Submitted on 1 Jan 1977

HAL is a multi-disciplinary open access archive for the deposit and dissemination of scientific research documents, whether they are published or not. The documents may come from teaching and research institutions in France or abroad, or from public or private research centers.
L'archive ouverte pluridisciplinaire HAL, est destinée au dépôt et à la diffusion de documents scientifiques de niveau recherche, publiés ou non, émanant des établissements d'enseignement et de recherche français ou étrangers, des laboratoires publics ou privés. 


\title{
Classification \\ Physics Abstracts \\ 6.550 \\ TUBES ÉLECTRONIQUES DE GRANDE PUISSANCE POUR Le CHAUfFage DeS Plasmas $(*)$
}

\author{
P. GUENARD et P. PALLUEL \\ Division Tubes Electroniques THOMSON-CSF, \\ 2, rue Latécoère, 78140 Velizy-Villacoublay, France
}

(Reçu le 30 décembre 1976, révisé le 22 avril 1977, accepté le 5 mai 1977)

\begin{abstract}
Résumé. - Certaines opérations, comme le chauffage des plasmas demandent, à des fréquences plus ou moins élevées, des sources de très grande puissance $(10 \mathrm{~kW}-1 \mathrm{MW})$. On examine les possibilités en puissance, en fonction de la fréquence, de divers types de tubes bien connus comme les triodes et tétrodes, klystrons et TOP, amplitron et, d'un développement nouveau, le gyrocone. Ces : tubes, pris dans leur ensemble, peuvent produire des puissances de $1 \mathrm{MW}$ ou plus jusqu'à environ $10 \mathrm{GHz}$. Au-delà, la chute en puissance est rapide, elle tombe en effet à $1 \mathrm{~kW}$ vers $100 \mathrm{GHz}$. On peut cependant repousser cette limite au-delà de $300 \mathrm{GHz}$ par l'emploi de tubes à résonance cyclotronique.
\end{abstract}

\begin{abstract}
In view of applications, such as plasma heating, which require, at more or less high frequencies, very high powers $(10 \mathrm{~kW}-1 \mathrm{MW})$ discussed in this paper are the power capabilities (as a function of frequency) of various well known types of tubes : triodes and tetrodes, klystrons, TWT's, and amplitrons ; and of a new development : the gyrocone. As a whole, these tubes can deliver powers of at least $1 \mathrm{MW}$ up to about $10 \mathrm{GHz}$. Beyond this frequency, the power drops down very rapidly and falls below $1 \mathrm{~kW}$ at $100 \mathrm{GHz}$. However, this limit may be pushed beyond $300 \mathrm{GHz}$ by using cyclotron resonance tubes.
\end{abstract}

1. Introduction. - Le chauffage des plasmas par l'application d'un signal alternatif de fréquence plus ou moins élevée, pouvant aller des fréquences les plus basses jusqu'au-delà de $300 \mathrm{GHz}$, requiert une puissance importante. Cette puissance peut atteindre ou dépasser $1 \mathrm{MW}$ dans un régime d'impulsions très longues dépassant largement $1 \mathrm{~ms}$, durée d'impulsion qui exige que les tubes soient conçus pour fonctionner en régime permanent.

Dans ce papier, on examinera donc les possibilités des tubes capables de fournir une très grande puissance, de quelques kilowatts à quelques mégawatts en régime permanent, que ce soient des tubes dérivés des modèles qui figurent actuellement dans les catalogues ou résultant de développements nouveaux.

De tels tubes ont déjà été développés dans le passé [1]: en 1960, dans le cadre d'un programme militaire de tubes de super-puissance aux Etats-Unis, et depuis, pour des applications diverses, liées par exemple au chauffage par pertes diélectriques, à certains accélérateurs ou aux anneaux de stockage.

Ces applications demandent en général une fré-

$\left(^{*}\right)$ Communication présentée au Congrès National de Physique des Plasmas, Paris, 6-10 décembre 1976. quence fixe, sans modulation de la puissance, conditions qui sont favorables au fonctionnement des tubes. Par contre, même sans tenir compte du coût de la puissance qu'ils absorbent, il est nécessaire que leur rendement soit aussi élevé que possible, pour faciliter les problèmes technologiques posés par la dissipation de puissance dans le tube et réduire le volume des équipements auxiliaires.

Ce qui suit est relatif à la puissance unitaire des tubes et n'exclut pas la possibilité de faire des générateurs de puissance supérieure en associant des tubes en parallèle.

2. Triodes et tétrodes. - La puissance de ces tubes a été augmentée en repoussant successivement les limitations apportées par leurs différents éléments (Fig. 1). Actuellement, les résultats obtenus sont basés sur l'emploi $[2,3]$ :

- de cathodes thoriées donnant une densité de courant qui peut atteindre $5 \mathrm{~A} / \mathrm{cm}^{2}$,

- de grilles en graphite pyrolytique donnant à la fois une dissipation thermique par rayonnement élevée et une bonne protection contre l'émission thermique et l'émission secondaire,

- d'un système de refroidissement de l'anode 
faisant intervenir de l'eau en phase vapeur, au moins dans le radiateur du tube lui-même, et permettant des dissipations atteignant $2 \mathrm{~kW} / \mathrm{cm}^{2}$.

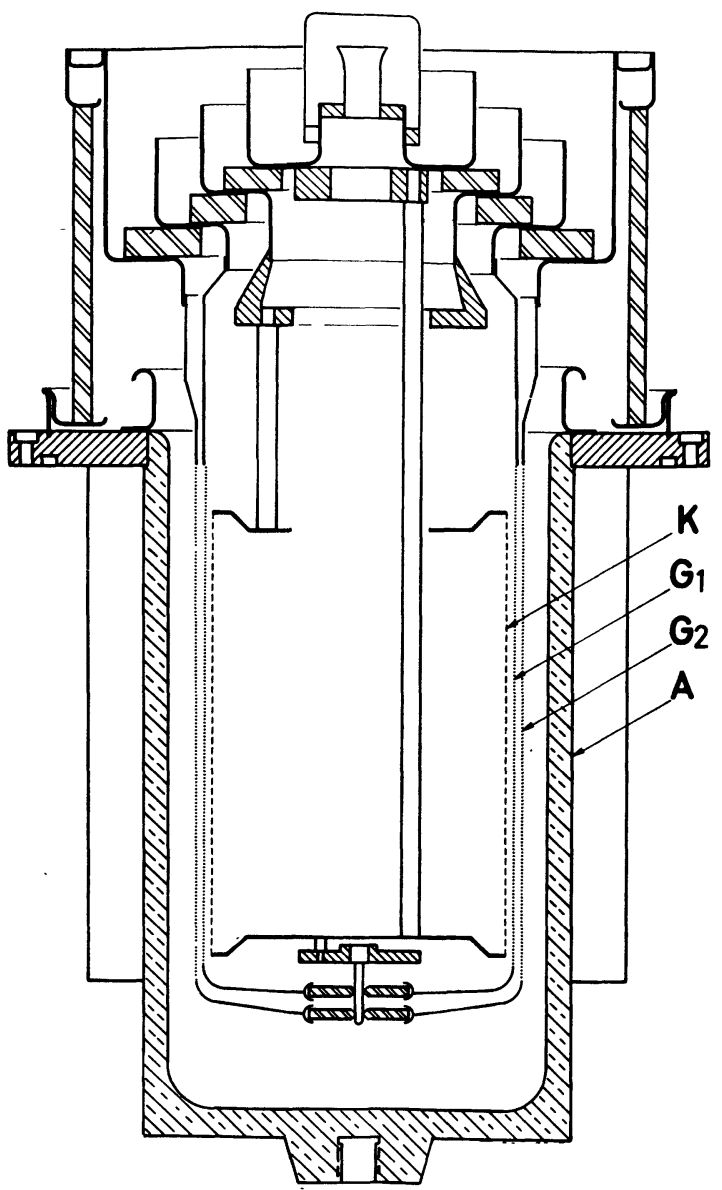

FIG. 1. - Coupe schématique d'une tétrode de grande puissance. $K$ cathode, $G_{1} G_{2}$ grilles, $A$ anode.

[Diagrammatic cross-section of a high-power tetrode. $\mathrm{K}=$ cathode, $\mathbf{G}_{1}, \mathbf{G}_{2}=$ grids, $A=$ anode.]

L'expérience montre que la tension anodique de ces tubes, pour des conditions favorables de fonctionnement permanent c'est-à-dire sans modulation, ne peut guère dépasser $20 \mathrm{kV}$. Au-delà, il apparaît des claquages généralement liés à des oscillations parasites. Pour des conditions difficiles telles que celles que l'on rencontre dans la radiodiffusion, cette limite s'abaisse vers $12 \mathrm{kV}$.

Toutes ces limitations conduisent à une puissance proportionnelle à la surface des électrodes. C'est effectivement ce qu'on observe en regardant les développements en cours. La figure 2 représente un tube de $500 \mathrm{~kW}$ couramment employé en radiodiffusion et la figure 3 les silhouettes de trois tubes :

- En $a$, tube donnant à $12 \mathrm{kV} \quad 0,5 \mathrm{MW}$ et à $20 \mathrm{kV} \quad 1 \mathrm{MW}$;

- En $b$, tube donnant à $12 \mathrm{kV} \quad 0,75 \mathrm{MW}$ et à $20 \mathrm{kV} \quad 1,5 \mathrm{MW}$;

- En $c$, tube donnant à $12 \mathrm{kV} 1 \mathrm{MW}$ et à $20 \mathrm{kV} 2 \mathrm{MW}$.

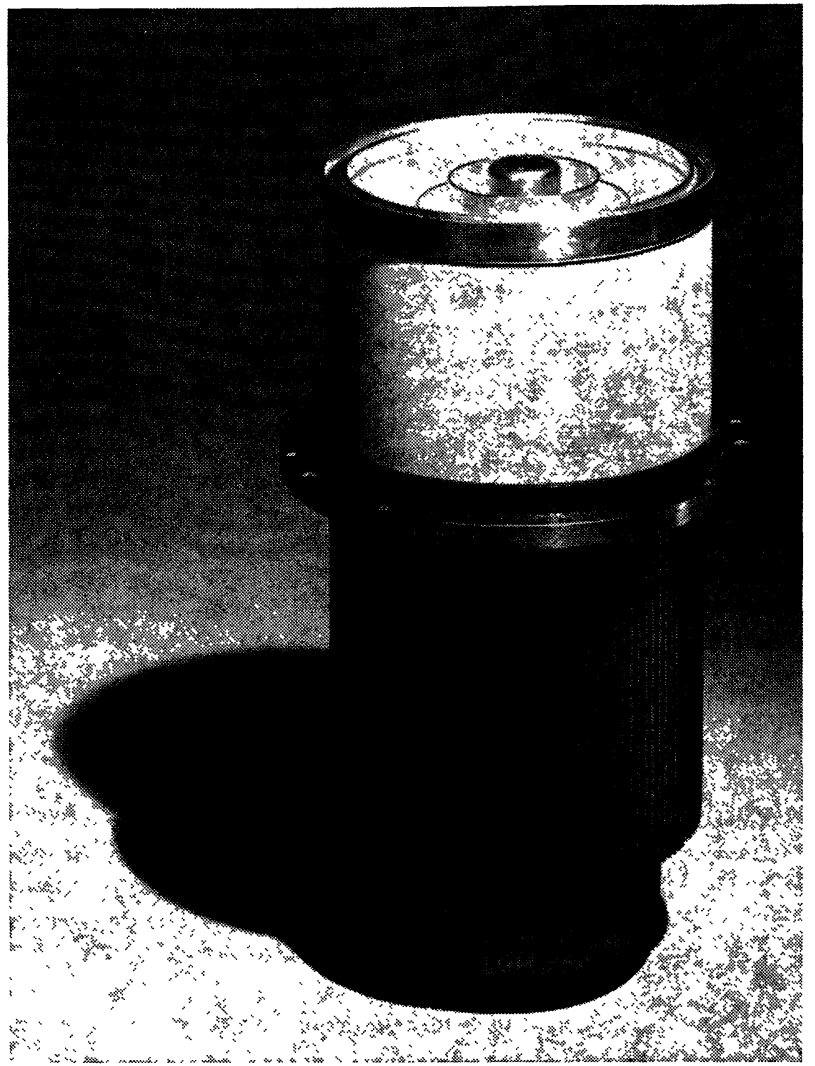

Fig. 2. - Tétrode TH 548 donnant $500 \mathrm{~kW}$ à $12 \mathrm{kV}$. [TH 548 Tetrode, delivering $500 \mathrm{~kW}$ at $12 \mathrm{kV}$.]

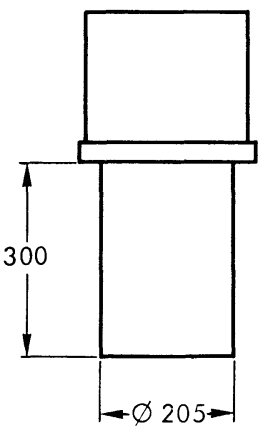

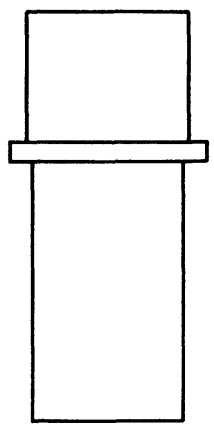

C

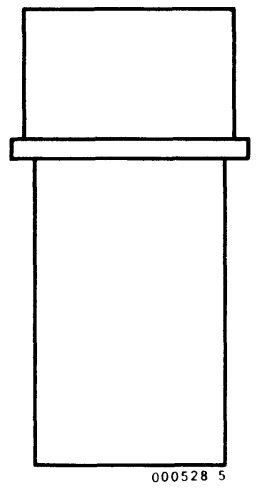

FIG. 3. - Silhouette de trois tubes de puissance croissante : a) TH 548, b) TH 558, c) $\mathrm{TH} 559$.

[Silhouette of three tubes in the order of increasing power : a) $\mathrm{TH} 548$, b) $\mathrm{TH} 558$, c) $\mathrm{TH} 559$.

Ce dernier tube en cours de développement montre donc qu'il est possible d'atteindre $2 \mathrm{MW}$ avec des tubes classiques. On peut se demander s'il est possible d'aller au-delà en continuant à augmenter la surface des électrodes. Intervient alors la fréquence limite de fonctionnement qui est d'autant plus basse que la capacité interélectrodes est plus élevée. Celle-ci est, aux capacités parasites près, proportionnelle à la surface des électrodes et donc à la puissance. On trouve ainsi une limitation de puissance inversement 
proportionnelle à la fréquence. Pour le tube de $2 \mathrm{MW}$, la fréquence limite est de $30 \mathrm{MHz}$. Dans cette zone de fréquences, le rendement des tubes est élevé. Il peut atteindre $90 \%$.

Lorsqu'on va vers des fréquences plus élevées, d'autres limitations apparaissent, pertes HF dans les diélectriques, qui donnent une chute proportionnelle au carré de la fréquence, puis limitations dues au temps de transit qui donnent une chute encore plus brutale. A $1 \mathrm{GHz}$, la puissance de ces tubes tombe à $10 \mathrm{~kW}$ et ils ne peuvent plus alors être utilisés dans les générateurs de très grande puissance. A ces fréquences très élevées, le rendement des tubes décroît d'ailleurs et rend leur utilisation moins intéressante vis-à-vis des tubes pour hyperfréquences.

3. Klystrons. - D'une fraction de $\mathrm{GHz}$ à quelques dizaines de $\mathrm{GHz}$, les générateurs de puissance actuels sont des tubes pour hyperfréquences. Parmi ces tubes, celui qui a donné les puissances les plus élevées est le klystron [4] (Fig. 4), favorisé en ce que la cathode qui fournit les électrons et le collecteur qui dissipe leur énergie résiduelle sont bien découplés de la

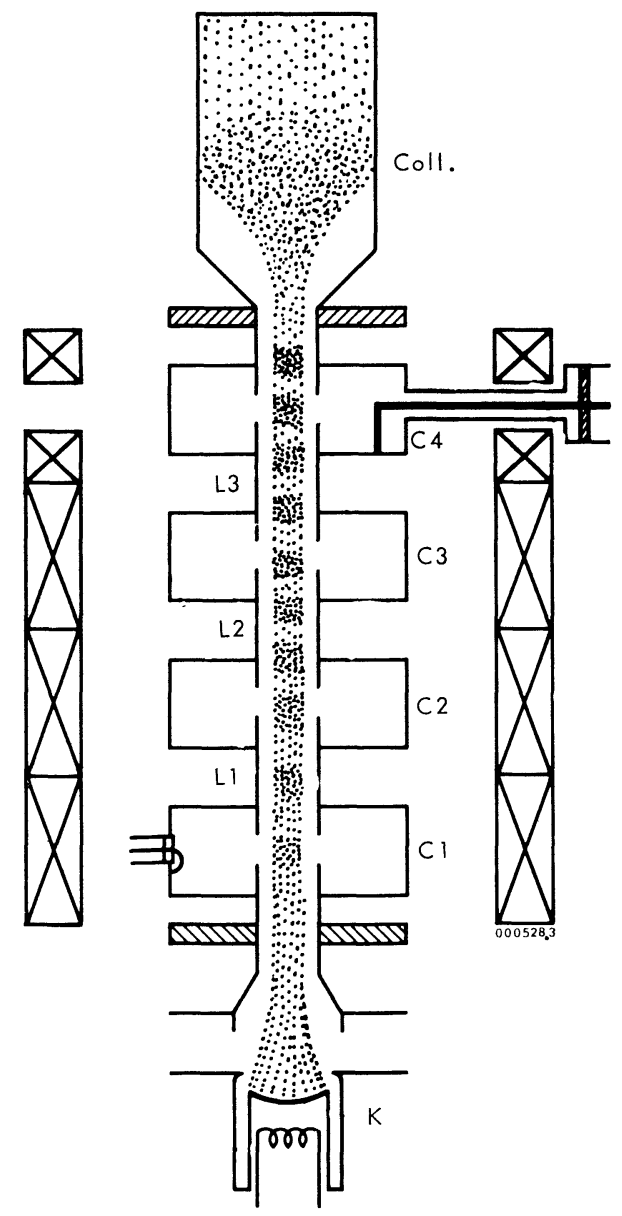

Fig. 4. - Coupe schématique d'un klystron. $\mathrm{K}$ cathode, Coll. collecteur, $C_{1} C_{2} C_{3} C_{4}$ cavités, $L_{1} L_{2} L_{3}$ espaces de glissement.

[Diagrammatic cross-section of a kystron. $\mathrm{K}=$ cathode ; Coll. $=$ collector ; $\mathrm{C}_{1}, \mathrm{C}_{2}, \mathrm{C}_{3}$ and $\mathrm{C}_{4}=$ cavities ; $\mathrm{L}_{1}, \mathrm{~L}_{2}$ and $\mathrm{L}_{3}=$ drift tubes.] structure hyperfréquence. Cette dernière, constituée d'une suite de cavités séparées ${ }_{\text {th }}$ par des espaces de glissement est thermiquement et mécaniquement robuste.

L'inconvénient de ce tube, au moins dans sa version originelle, pour la production de très grandes puissances, est son rendement relativement faible : 45-50\%. Des efforts ont été faits pour l'améliorer; et par l'emploi soit d'un espace de glissement allongé, soit de cavités harmoniques, on peut aller nettement au-delà. La figure 5 montre un tube à espace de glissement allongé destiné à l'alimentation d'un microtron et donnant un rendement de $62 \%$ [5].

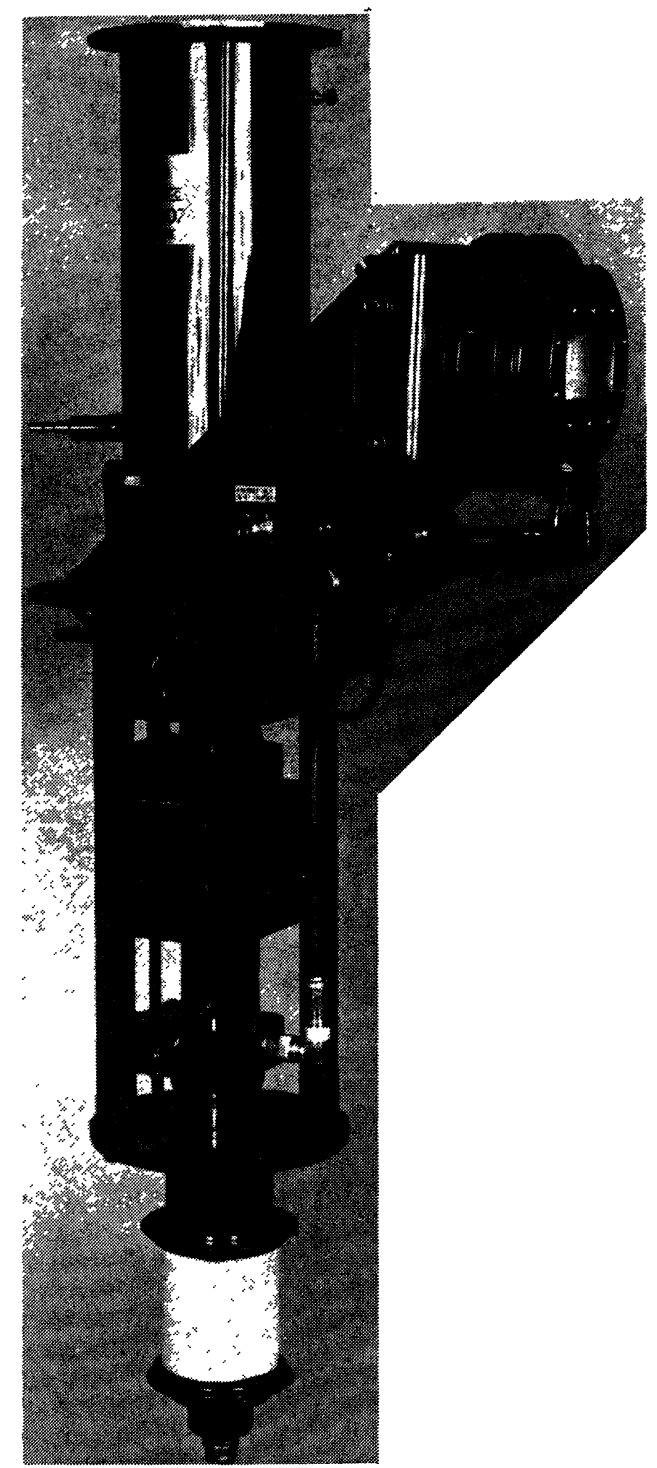

Fig. 5. - Tube TH 2075 à espace de glissement allongé donnant $50 \mathrm{~kW}$ avec $62 \%$ de rendement.

[TH 2075 klystron with elongated drift tube, delivering $50 \mathrm{~kW}$ of output power with an efficiency of $62 \%$.]

Cette augmentation du rendement n'est cependant efficace que si la pervéance $\left(\mathfrak{T}=I_{0} V_{0}^{-3 / 2}\right)$ n'est pas élevée et si d'autre part le rayon $a$ du trou de 
passage du faisceau dans les cavités est petit $\left(\omega a / v_{0}=0,5\right)$ comme le montre la figure 6 qui reproduit les résultats de deux calculs théoriques et place quelques points expérimentaux. Cette figure fait apparaître des rendements possibles dépassant $70 \%[5,6,7,8]$.

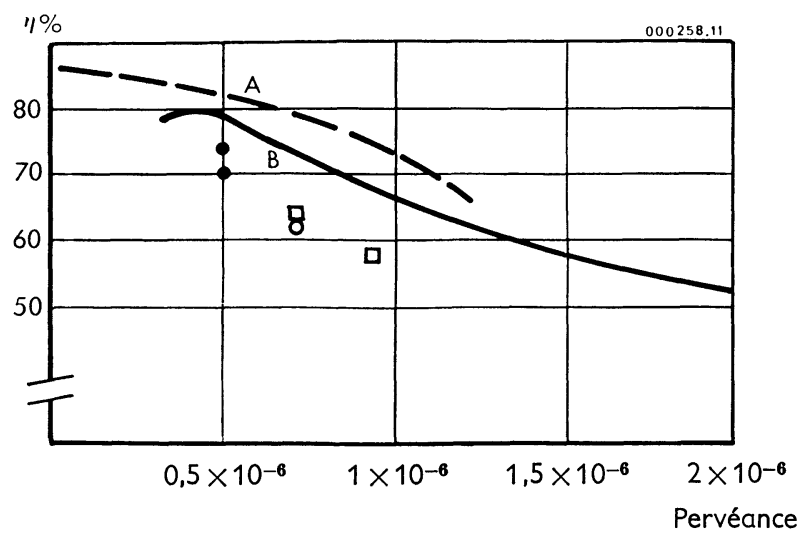

FIG. 6. - Variation du rendement avec la pervéance. A et B courbes calculées d'après [5] et [6]. Points de mesure : $\bigcirc$ [5], - [7], $\square[8]$.

[Variation of efficiency with perveance. Curves A and B computed in accordance with [5] and [6]. Measured points : $\bigcirc$ [5], [7], $\square[8]$.

Les limitations de la puissance d'un klystron viennent en premier lieu :

1) De la densité de courant sur la cathode $J_{\mathbf{k}}$, de la convergence du canon $C$, du rayon du faisceau $b$ (qui intervient par $b^{\prime}=\omega b / v_{0}$ ) qui conduisent, en fonction de la fréquence, aux expressions du courant :

$$
\begin{aligned}
& I_{0}=\frac{e}{2 \pi m} \frac{b^{\prime 2} J_{\mathrm{k}} C}{f^{2}} V_{0} \quad \text { (non relativiste) } \\
& I_{0}=\frac{c^{2}}{4 \pi} \frac{b^{\prime 2} J_{\mathrm{k}} C}{f^{2}} \quad \text { (très relativiste) }
\end{aligned}
$$

de bonnes conditions pour la focalisation, la durée de vie et le rendement sont par exemple :

$$
J_{\mathrm{k}}=1,5 \mathrm{~A} / \mathrm{cm}^{2} \quad C=25 \quad b^{\prime 2}=0,1 ;
$$

de mauvaises conditions sont par exemple :

$$
J_{\mathrm{k}}=5 \mathrm{~A} / \mathrm{cm}^{2} \quad C=50 \quad b^{\prime 2}=1 .
$$

2) Des pertes hyperfréquences dans la cavité de sortie qui réduisent le rendement. A la résonance, cette cavité présente une impédance $R$ de l'ordre de $1000 / f^{1 / 2} \mathrm{k} \Omega$, où la fréquence $f$ est mesurée en $\mathrm{GHz}$. L'impédance de charge totale de la cavité étant de l'ordre de $V_{0} / I_{0}$, de bonnes conditions correspondent à :

$$
\frac{V_{0}}{I_{0}}=\frac{R}{10}
$$

qui abaisse le rendement du dixième de sa valeur; de mauvaises conditions à :

$$
\frac{V_{0}}{I_{0}}=\frac{R}{2}
$$

qui divise le rendement par 2.

Les figures 7, 9, 10 montrent ce qui se passe à $1 \mathrm{GHz}, 10 \mathrm{GHz}$ et $30 \mathrm{GHz}$.

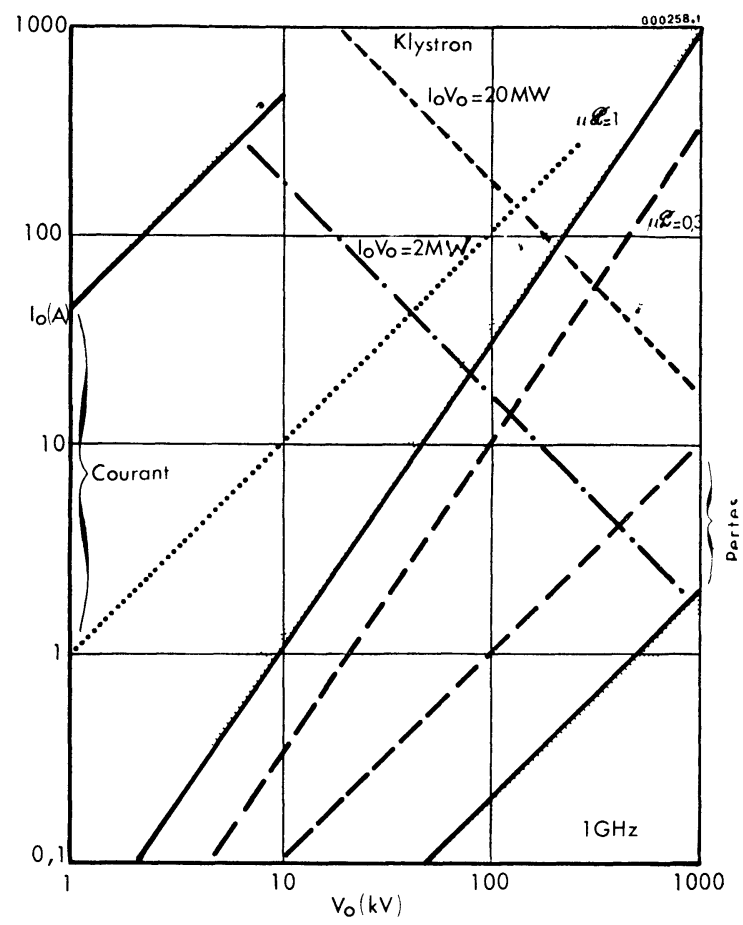

Fig: 7. - Diagramme de discussion de l'influence des paramètres du klystron sur son fonctionnement à $1 \mathrm{GHz}$ : . . . . . bonnes conditions pour le courant, - - - - - bonnes conditions pour les pertes, 1 mauvaises conditions au-delà de cette limite.

[Diagram for discussing the influence of klystron parameters on its operating at $1 \mathrm{GHz}$ :..... . good conditions for the current, - - - - - - good conditions for the losses, \%- conditions beyond this limit.

A $1 \mathrm{GHz}$ et en dessous toutes les bonnes conditions sont simultanément réalisées, la puissance que l'on peut obtenir sera alors limitée par d'autres considérations (par exemple claquage et dissipation thermique) qui font varier la puissance en $1 / f$. Elle dépasse de toute façon largement $1 \mathrm{MW}$ et on peut atteindre le maximum de rendement, à condition de ne pas imposer d'autres conditions telles qu'une valeur maximale de la tension qui oblige à augmenter la pervéance et à s'écarter des conditions de bon rendement. Aux basses fréquences, le tube devient volumineux (Fig. 8).

A $10 \mathrm{GHz}$ (Fig. 9), on ne peut plus avoir simultanément toutes les conditions de bon fonctionnement, on peut cependant encore atteindre $1 \mathrm{MW}$. Une difficulté vient de la puissance perdue dans la cavité de sortie, puissance qui est de l'ordre de $20 \mathrm{~kW}$. 


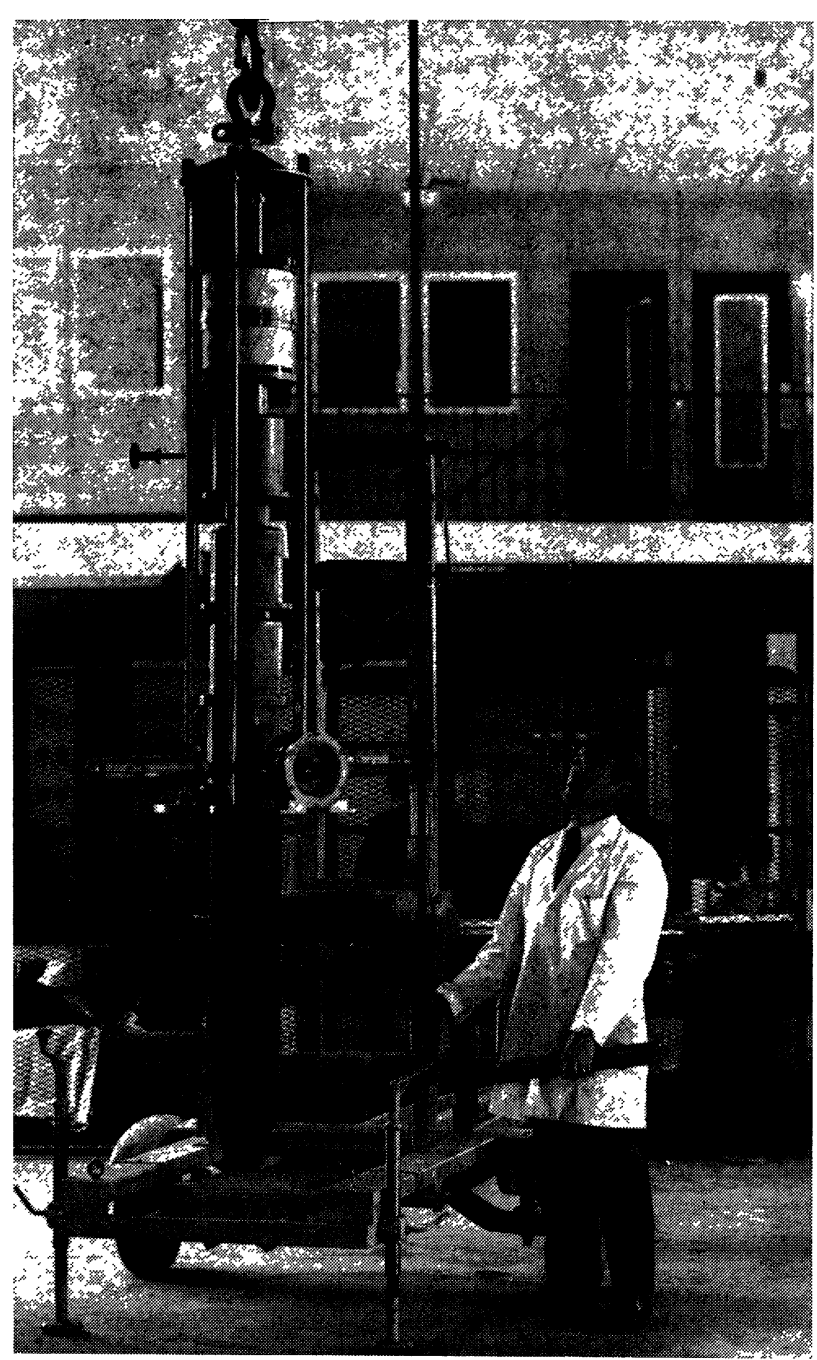

Fig. 8. - Klystron F 2055 pouvant donner $500 \mathrm{MW}$ à $500 \mathrm{kHz}$. [F 2055 klystron : capable of delivering $500 \mathrm{MW}$ at $500 \mathrm{kHz}$.]

A $30 \mathrm{GHz}$ (Fig. 10), il reste une zone possible à faible puissance. On peut espérer atteindre quelques kilowatts avec un rendement faible (10 à $15 \%$ ).

On peut repousser la limitation due aux pertes hyperfréquences dans la cavité de sortie, pertes qui diminuent le rendement et posent des problèmes de dissipation thermique en utilisant pour le prélèvement d'énergie une interaction prolongée telle que celle qui existe dans les tubes à ondes progressives et repousser la limite de $1 \mathrm{~kW}$ au-delà de $100 \mathrm{GHz}$. Les autres limitations subsistent cependant et les klystrons ou les tubes à ondes progressives n'offrent pas actuellement de solution au problème de la très grande puissance au-delà d'environ $20 \mathrm{GHz}$.

4. Amplitron. - Cet amplificateur à ondes régressives à champs croisés présente l'intérêt d'un rendement élevé, qui peut atteindre $80 \%$, l'inconvénient que son circuit à retard doit dissiper l'énergie résiduelle du faisceau. En constituant les éléments de la ligne à retard (Fig. 11) par des faisceaux de tubes, parcourus par un courant d'eau à grande vitesse et

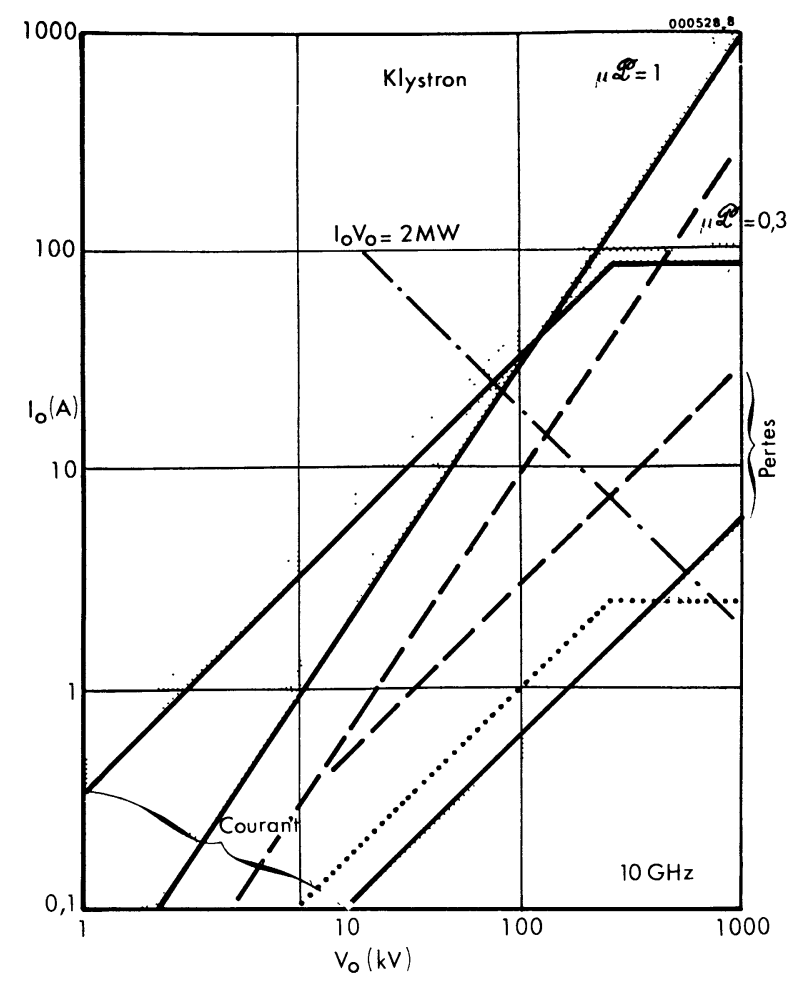

Fig. 9. - Diagramme de discussion du klystron à $10 \mathrm{GHz}$.

[Diagram for discussing a $10 \mathrm{GHz}$ klystron.]

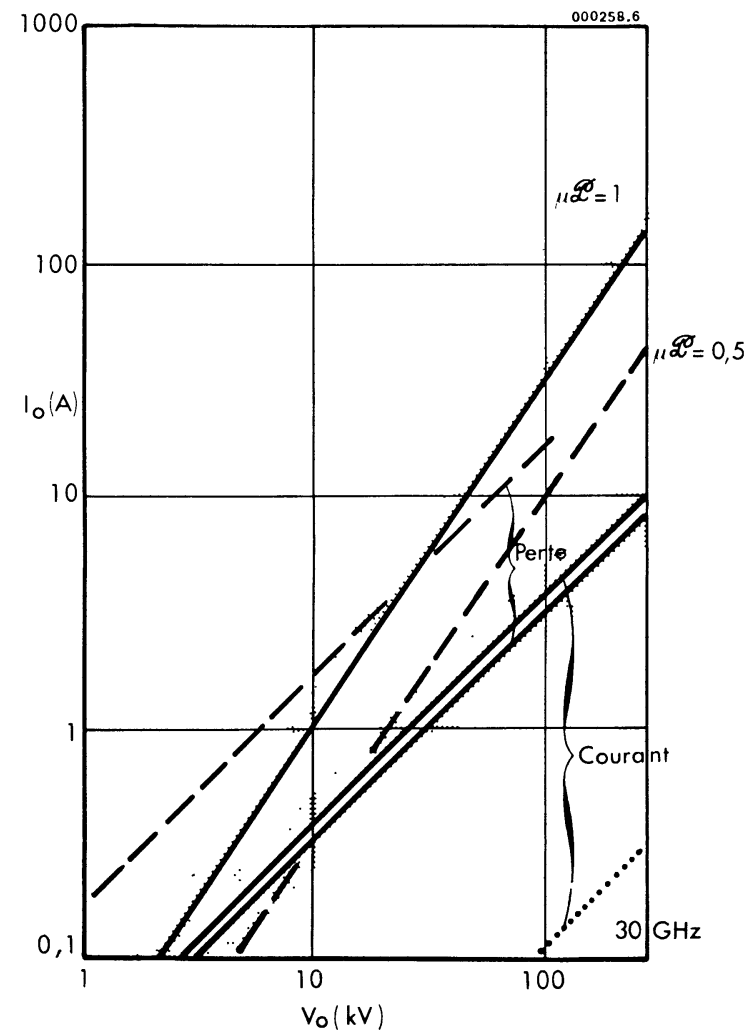

FIG. 10. - Diagramme de discussion du klystron à $30 \mathrm{GHz}$.

[Diagram for discussing a $30 \mathrm{GHz}$ klystron.]

torsadés pour éviter que le même tube n'ait à dissiper sur toute sa longueur, on a obtenu une puissance de plus de $400 \mathrm{~kW}$ à $3 \mathrm{GHz}$ à $72 \%$ de rendement [9]. 


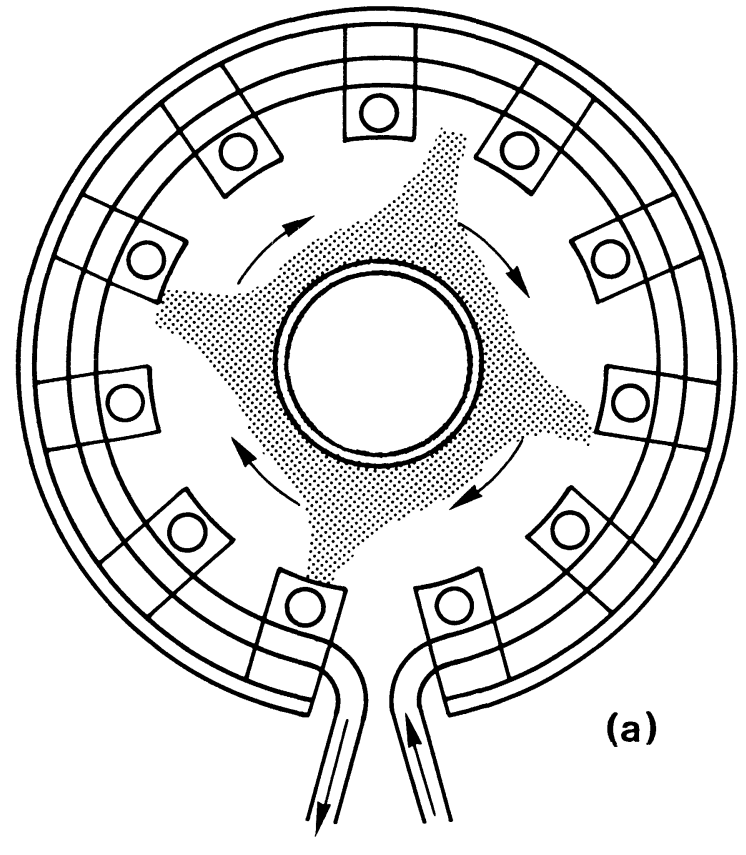

(b)

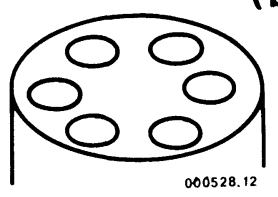

Fig. 11. - Coupe schématique : a) de l'amplitron, $b$ ) d'un élément de circuit.

[Schematic cross-section of $a$ ) the amplitron, $b$ ) a circuit element. ]

Ceci a été en fait réalisé avec deux tubes en série donnant ensemble un gain d'environ $10 \mathrm{~dB}$, à une tension anodique assez basse $20 \mathrm{kV}$. A des fréquences plus basses, et ne tenant compte que de la limitation thermique, qui prévoit une puissance variant comme l'inverse de la fréquence, on pourrait ainsi obtenir avec ce type de tube plus de $1 \mathrm{MW}$ à une fréquence de $1 \mathrm{GHz}$.

5. Gyrocone. - Ce tube proposé par Budker [10] est basé sur la rotation d'un faisceau électronique initialement vertical, sur un cône d'angle faible $\left(5^{\circ}\right)$ sous l'action d'un champ magnétique tournant. Celui-ci apparaît au centre d'une cavité de mode $\mathrm{E}_{110}$, alimentée par deux sources en quadrature de phase et résonnant à la fréquence de fonctionnement (Fig. 12). Le faisceau est ensuite dévié vers l'horizontale par le champ magnétique d'une bobine conique et traverse un résonateur torique où il excite une onde progressive qui le freine (Fig. 13). Ce dispositif qui ne fait pas appel à un groupement en paquet ou en

(1) Ces informations nous ont été aimablement communiquées par M. Zyngier du Service Anneaux de Collision d'Orsay.

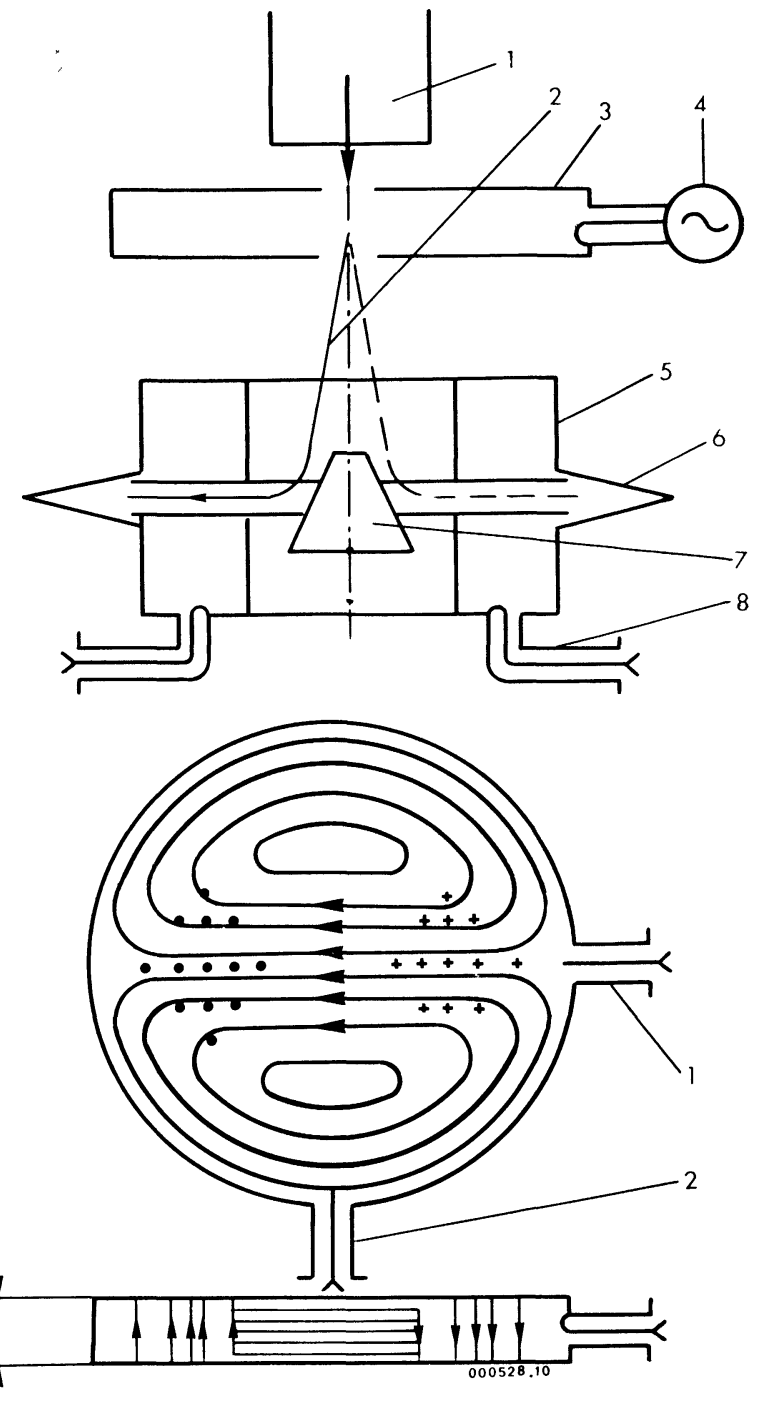

Fig. 12. - Coupe schématique du gyrocone et champs dans la cavité d'entrée (d'après [10]). 1 . Source de courant ; 2 . Faisceau d'électrons ; 3. Cavité d'entrée ; 4. Lignes d'excitation de la cavité d'entrée ; 5 . Cavité de sortie ; 6 . Collecteur ; 7. Bobine de déviation; 8. Sortie de puissance.

[Schematic cross-section of the gyrocone and fields in the input cavity (after [10]). 1. Current source; 2. Electron beam; 3. Input cavity; 4. Excitation lines for the input cavity; 5. Output cavity ; 6. Collector ; 7. Deflection coil ; 8. Power output.]

phase des électrons permet d'obtenir un rendement voisin de $100 \%$, les électrons pouvant être tous également freinés. Les estimations actuelles faites sur un modèle fonctionnant avec un faisceau de 12 A sous $500 \mathrm{kV}$ sont un rendement électronique de $95 \%$, un rendement total (y compris les dispositifs de stabilisation de la tension) de $80 \%$, ceci à $180 \mathrm{MHz}$.

Le point critique de ce tube est son optique électronique. En supposant que le projet actuel corresponde à un optimum dans ce domaine, il ne faut donc pas, lorsqu'on augmente la fréquence et qu'on réduit proportionnellement les dimensions, augmenter la pervéance du faisceau. La densité de courant 


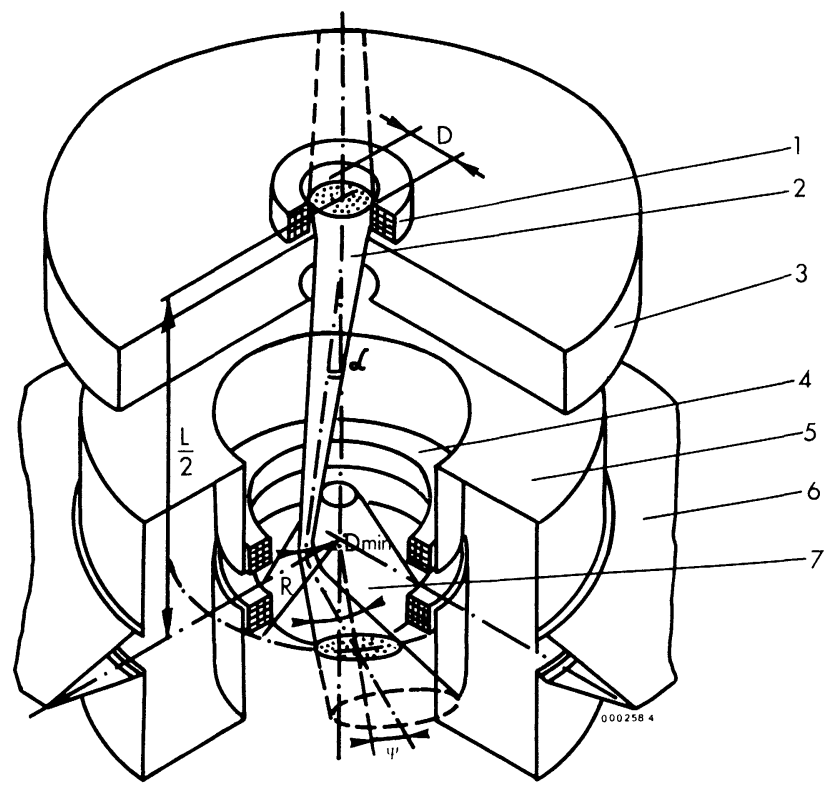

Fig. 13. - Vue en perspective du gyrocone (d'après [10]). 1. Bobine de concentration ; 2. Faisceau électronique ; 3. Cavité d'entrée; 4. Bobines de focalisation cylindrique; 5. Cavité de sortie ; 6. Collecteur ; 7. Bobine de déflexion.

[Perspective view of the gyrocone (after [10]). 1. Concentration coil ; 2 . Electron beam ; 3. Input cavity ; 4. Cylindrical-focusing coils ; 5 . Output cavity ; 6. Collector ; 7. Deflection coil.]

a d'autre part une valeur limite qui à partir d'une certaine fréquence impose une décroissance du courant comme $f^{-2}$. Enfin, la résistance parallèle de pertes de la cavité, en se basant sur une puissance

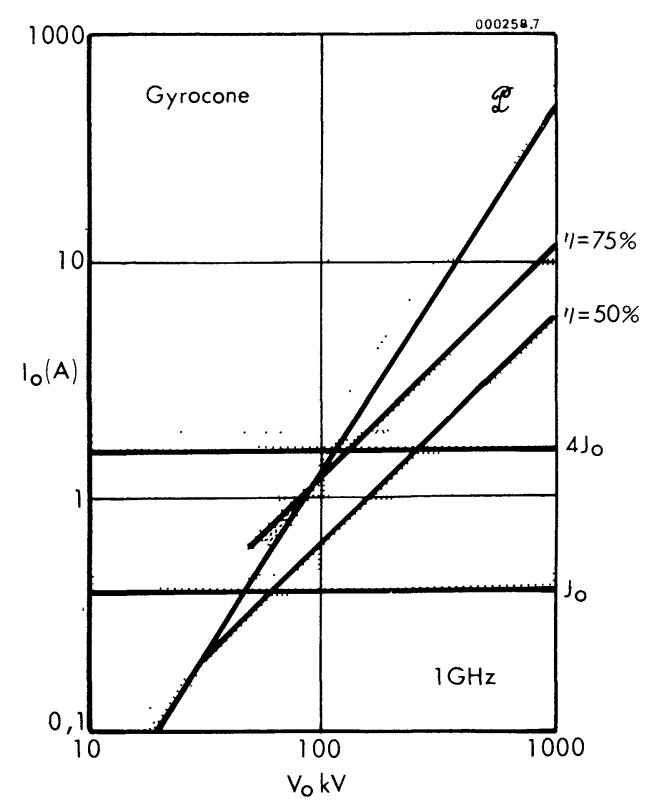

FIG. 14. - Diagramme de discussion du gyrocone. ㅃ..: Limites à ne pas dépasser pour obtenir le courant, le rendement ou la pervéance.

[Diagram for discussing the gyrocone. exceeded to obtain the current, the efficiency or the perveance.] de pertes de $200 \mathrm{~kW}$ dans le projet actuel à $0,18 \mathrm{GHz}$, est approximativement de la forme :

$$
R=600\left(\frac{0,18}{f}\right)^{1 / 2} \mathrm{k} \Omega .
$$

Ceci conduit pour $f=1 \mathrm{GHz}$ à la discussion résumée dans la figure 14, où on a admis deux valeurs de la densité de courant, celle du projet actuel et une densité quatre fois plus grande. Cette figure montre que dans la première hypothèse la puissance possible ne dépasse guère $10 \mathrm{~kW}$ avec un rendement de $50 \%$. Dans la deuxième hypothèse, la puissance peut atteindre $100 \mathrm{~kW}$ avec un rendement de $75 \%$.

6. Conclusion. - La figure 15 résume cette discussion. Aux basses fréquences, $f<0,03 \mathrm{GHz}$, les triodes et tétrodes fournissent des puissances supé-

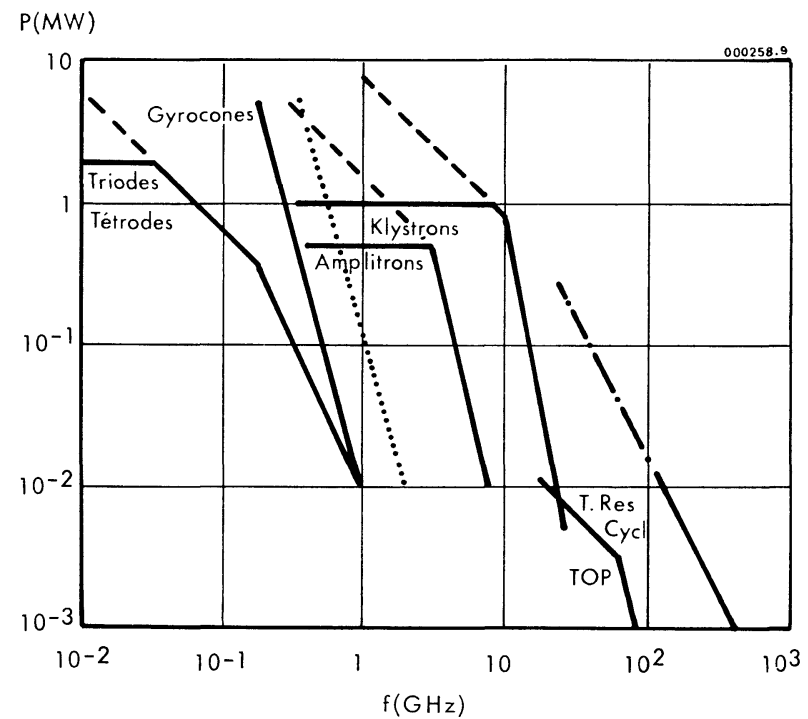

FIG. 15. - Puissance que l'on peut obtenir avec divers modèles de tubes. En trait plein, puissance obtenue (espérée pour le gyrocone). Pour les parties horizontales de ces courbes la puissance a été au moins obtenue à la fréquence la plus élevée. En trait interrompu, prolongement en $1 / f$ à partir de ce point. En pointillé, puissance que l'on pourrait attendre du gyrocone en multipliant par 4 la densité de courant. En trait mixte, prolongement des tubes à résonance cyclotronique suivant des développements annoncés.

[Power that can be obtained with different types of electron tubes. In solid lines, power obtained (anticipated for the gyrocone). For the horizontal parts of these curves, the power has at least been obtained at the highest frequency. The dashed lines show the continuation by $1 / f$ from this point. The dotted line shows the power that could be expected from the gyrocone by multiplying the current density by 4 . The dot-dash line shows the extension of the power from cyclotronic resonance tubes according to the developments announced.]

rieures à $1 \mathrm{MW}$. Ces puissances décroissent ensuite et tombent à $0,01 \mathrm{MW}$ à $1 \mathrm{GHz}$, avec les structures actuelles. Dans cette zone de fréquences des structures différentes, telles que celle utilisée autrefois sur le resnatron, peuvent conduire à des puissances deux à trois fois supérieures. 
Ensuite apparaissent le gyrocone, l'amplitron et le klystron. Dans la zone de 0,15 à $1 \mathrm{GHz}$, ces trois tubes peuvent être réalisés, le choix entre eux dépendra des caractéristiques particulières demandées et de l'état de leur développement dans un domaine de fréquence et de puissance voisines. Vers $0,5-0,8 \mathrm{GHz}$ l'intérêt du gyrocone, tel qu'il est conçu actuellement, disparaît vis-à-vis des deux autres tubes, et au-delà de $3 \mathrm{GHz}$ l'intérêt de l'amplitron disparaît vis-à-vis de celui du klystron. Au-delà de $10 \mathrm{GHz}$, la puissance du klystron diminue rapidement et malgré une certaine extension en fréquences à des puissances modestes $(1-10 \mathrm{~kW})$ due au tube à ondes progressives, les modèles actuels de tubes pour hyperfréquences sont incapables de fournir de très grandes puissances. Les seuls dispositifs actuellement étudiés qui per- mettent d'aller au-delà sont les tubes à résonance cyclotronique $\left({ }^{2}\right)$, qui actuellement réalisés au-delà de $150 \mathrm{GHz}$, peuvent vraisemblablement donner des puissances élevées (supérieures à $100 \mathrm{~kW}$ ) vers 20 $40 \mathrm{GHz}$.

Les valeurs des puissances indiquées sur la figure 15 correspondent aux limites actuelles des types de tubes mentionnés et ne tiennent aucun compte d'autres considérations par exemple le problème des alimentations ou celui du coût et du délai de leur développement. Toutes ces considérations, y compris la fiabilité des tubes conduiront souvent à se placer en deçà de cette limite.

(2) Ces tubes sont examinés dans la communication de R. Le Gardeur présentée au même congrès.

\section{Bibliographie}

[1] OKRess, E. C., Microwave Power Engineering (Academic Press) 1968.

[2] Agniel, R. and Turteltaub, M., International Broadcasting Convention, Londres (Sept. 1974).

[3] Gerlach, P., Fachtagung Hörrundfunk (Düsseldorf), nov. 1976.

[4] Luebke, W., Caryotakis, G., Microwave J. 9 (1966) 41.

[5] Mihran, T. G., Branch, G. M., Jr., Griffin, G. J., Jr., IEEE Trans. Electron Devices 18 (1971) 124.

[6] Kosmahl, H. G., Albers, L. U., IEEE Trans. Electron Devices 20 (1973) 883.
[7] LIEN, E. L., Proceedings of the Eighth International Conference on Microwaves and Optical Generation and Amplification, Amsterdam (Sept. 1970) Klüwer-Deventer, p. 11-21 à 11-27.

[8] Maloney, E. D. and Faillon, G., J. Microwave Power 9 (1974) 231

[9] Skowron, J. F., Brown, W. C., Mac Master, G. H., Microwave J. 7 (1964) 65.

[10] Budker, G. et al., Communication au $\mathrm{V}^{\mathrm{e}}$ Congrès soviétique sur les accélérateurs. 